\title{
Modeling the Color Image and Video Quality on Liquid Crystal Displays with Backlight Dimming
}

\author{
Korhonen, Jari; Mantel, Claire; Burini, Nino; Forchhammer, Søren
}

Published in:

Proceedings of International Conference on Visual Communications and Image Processing (VCIP'13)

Link to article, DOI:

10.1109/VCIP.2013.6706383

Publication date:

2013

Link back to DTU Orbit

Citation $(A P A)$ :

Korhonen, J., Mantel, C., Burini, N., \& Forchhammer, S. (2013). Modeling the Color Image and Video Quality on Liquid Crystal Displays with Backlight Dimming. In Proceedings of International Conference on Visual Communications and Image Processing (VCIP'13) IEEE. https://doi.org/10.1109/VCIP.2013.6706383

\section{General rights}

Copyright and moral rights for the publications made accessible in the public portal are retained by the authors and/or other copyright owners and it is a condition of accessing publications that users recognise and abide by the legal requirements associated with these rights.

- Users may download and print one copy of any publication from the public portal for the purpose of private study or research.

- You may not further distribute the material or use it for any profit-making activity or commercial gain

- You may freely distribute the URL identifying the publication in the public portal 


\title{
MODELING THE COLOR IMAGE AND VIDEO QUALITY ON LIQUID CRYSTAL DISPLAYS WITH BACKLIGHT DIMMING
}

\author{
Jari Korhonen, Claire Mantel, Nino Burini, and Søren Forchhammer \\ Dept. of Photonics Engineering, Technical University of Denmark (DTU Fotonik)
}

\begin{abstract}
Objective image and video quality metrics focus mostly on the digital representation of the signal. However, the display characteristics are also essential for the overall Quality of Experience (QoE). In this paper, we use a model of a backlight dimming system for Liquid Crystal Display (LCD) and show how the modeled image can be used as an input to quality assessment algorithms. For quality assessment, we propose an image quality metric, based on Peak Signal-to-Noise Ratio (PSNR) computation in the CIE $\mathrm{L} * \mathrm{a} * \mathrm{~b} *$ color space. The metric takes luminance reduction, color distortion and loss of uniformity in the resulting image in consideration. Subjective evaluations of images generated using different backlight dimming algorithms and clipping strategies show that the proposed metric estimates the perceived image quality more accurately than conventional PSNR.
\end{abstract}

Index Terms - Video quality assessment, Liquid crystal display, Local backlight dimming

\section{INTRODUCTION}

Since subjective quality assessment performed by human test subjects is often very time and resource consuming, several different objective (i.e. algorithm based) metrics have been developed and proposed for evaluating compression and transmission artifacts in digital images and video sequences. These methods are useful for rapid experimentation and comparison between different artifacts introduced in the digital signal. However, the actual quality perceived by the end user is also highly dependent on the display device. Low contrast, distorted color representation or blurriness on the display may disturb the experienced quality, even if the digital input signal is of perfect quality.

Nowadays, LCDs are widely used in computer monitors and TV sets. LCD technology is based on voltage controlled liquid crystal pixels working as shutter elements, blocking the backlight and in this way rendering the desired brightness level. In color displays, pixels are formed of red, green and blue $(\mathrm{R}, \mathrm{G}, \mathrm{B})$ subpixels. The full range of possible colors is produced as a combination of $\mathrm{R}, \mathrm{G}$ and $\mathrm{B}$ components. In practical LCDs, a large amount of light is dissipated by the screen elements and only a fraction of it reaches the viewer [1]. This is why a bright backlight is typically needed, which leads to high power consumption in LCDs. Another disadvantage of LCD is light leakage: LC elements cannot block all the light even for the entirely black pixels, so some light leaks through them, making dark areas of the image look slightly grayish. Light leakage is visible in particular from large viewing angles.

In order to alleviate the problem of high power consumption and light leakage, different local backlight dimming schemes have been proposed [2-5]. The basic idea of local backlight dimming is simple: instead of using a uniform backlight over the whole display, there are several backlight segments, often using Light Emitting Diodes (LED) as light sources, that can be adjusted separately. LEDs may be located behind the LCs (direct-lit backlight) or at the edges of the display (edge-lit backlight). The transmittance of the LC pixels can be increased to compensate the reduced backlight. Backlight dimming allows using lower light intensity in the dark areas of the image, and therefore reduces power consumption and local light leakage. Between backlight elements and LCs, there is a diffuser plate, that mixes the light coming from individual elements.

Backlight dimming often involves decreased brightness of some pixels, causing a defect called clipping. Leakage and clipping both compromise the image quality. For the clipping part, quality is basically a trade-off with power consumption [6]; dimming reduces power consumption, but results in more severe clipping effects. In order to model the physical image rendered by the display, the backlight intensity needs to be modeled at each pixel position on the diffuser plate, and then combined with the pixel transmittance at the respective LC element [7].

Traditional image distortion measures, such as PSNR, have not been designed for backlight dimming artifacts. In this paper, we propose a new yet simple metric, based on PSNR in CIE L*a*b* color space, taking clipping effect and color distortion into consideration better than conventional PSNR. Modeling a backlight dimming display is discussed more comprehensively in Section 2. In Section 3, the proposed metric and its use within the backlight dimming model is explained. Section 4 summarizes simulation results comparing the proposed metric against several conventional metrics in respect to results of subjective quality assessment. Finally, concluding remarks are given in Section 5. 


\section{MODEL OF A BACKLIGHT DIMMING DISPLAY}

There are several different aspects that need to be taken into consideration when modeling a local backlight dimming system. In a basic model, we only consider normalized backlight intensities and LC transmittance levels, light distribution on the diffuser plate and light leakage. In a more advanced model, we add some more sophisticated features, such as the connection between physical and perceived intensity, and different pixel compensation schemes.

\subsection{Basic model}

A generic local backlight dimming algorithm takes a digital image as input and gives the intensities for each backlight segment and the transmittance of LC pixels as output. The observed luminance $L$ at pixel $(i, j)$ can be calculated by multiplying the backlight intensity $B$ at the pixel with the LC transmittance $T$ of the pixel:

$$
L(i, j)=B(i, j) T(i, j),
$$

where $L, B$ and $T$ are normalized to the interval $[0,1]$, where 0 is no light and 1 is full light intensity.

However, Eq. (1) does not consider the impact of light leakage. Assuming full backlight and zero transmittance $(B=1, T=0)$, the observed luminance would optimally be $L=0$. Due to light leakage, this is not the case, since the leaked light is observed. To model this aspect, a leakage factor $\varepsilon$ is defined [5]. In practice, $\varepsilon$ depends on the viewing angle and may vary between pixels and color components. In a simple model, constant $\varepsilon$ may be assumed for the whole display. The relationship between observed and ideal luminance with backlight levels $B=0.5$ and $B=1$ is illustrated in Fig. 1., and the normalized luminance with the leakage is given by:

$$
L(i, j)=B(i, j) T(i, j)+\varepsilon(i, j) B(i, j)(1-T(i, j))
$$

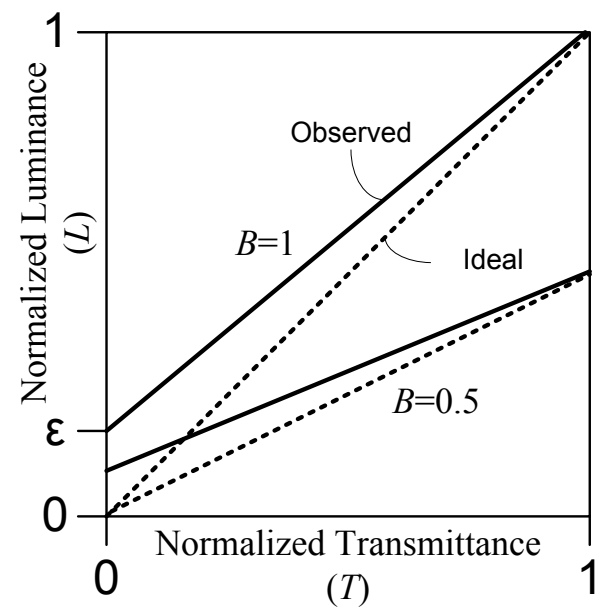

Fig. 1. Impact of light leakage to observed luminance.
In practical LCDs, light from different backlight units is diffused and mixed on the diffuser plate located between the light source and the liquid crystals. Light diffusion can be modeled with a Point Spread Function (PSF) $h_{k}: h_{k}(i, j)$ is the amount of light from backlight unit $k$ reaching the pixel at position $(i, j)$. Then, the normalized intensity at $(i, j)$ is $h_{k}(i, j)$ multiplied by the intensity of backlight element $k$, denoted as $B_{k}$. Since the backlight intensity at each position $B(i, j)$ may be contributed by all the backlight units $k$, the actual intensity can be computed as a sum of all $N$ contributions:

$$
B(i, j)=\sum_{k=1 . . N} B_{k} h_{k}(i, j)
$$

Equation (3) allows different PSFs for each backlight unit. However, in practical applications with direct-lit backlight, similar PSF is often used, centered around the midpoint of the backlight element. To get the most accurate results, PSF should be measured for each device independently. However, Gaussian PSF may be used as a simplified approximation of PSF in direct-lit backlights [7].

\subsection{Perceptually uniform vs. physical luminance}

Digital images and video sequences are ideally presented in a perceptually uniform format, i.e. certain intervals in pixel values represent similar differences in perceived intensity over the whole applicable range. In particular, sRGB is an approximately uniform representation of the color components [8]. Unfortunately, the relationship between physical luminance and perceived brightness is not linear: increasing physical luminance by a certain step size is perceptually more noticeable in the dark than in the bright part of the luminance range. This is why practical displays usually perform gamma correction when the input image is converted to LC transmittance. Denoting normalized perceived (uniform) luminance $L_{U}$ and physical luminance $L$, Gamma and inverse Gamma corrections are defined as:

$$
\left\{\begin{array}{c}
L=L_{U}^{\gamma} \\
L_{U}=L^{1 / \gamma}
\end{array}\right.
$$

A typical value of $\gamma$ is 2.2 , but other values around 2 are also used [9]. It should be noted that in practical LCDs, input signal controlling the backlights is usually directly proportional to the physical luminance, whereas the input signal to LC is in sRGB, that is then Gamma corrected by the display hardware. In the Eqs. (1) and (2), $L, B$, and $T$ are supposed to be expressed in the physical domain. This is why the input values must be converted to the same domain before computations, in our case by applying inverse Gamma correction to $T$.

It has been reported that the optimal $\gamma$ is often different for R, G and B components of a color signal [9]. We have omitted this aspect in our study; however, it would be possible to apply different Gamma correction to color components. In general, Gamma function has shown to 
work well with conventional displays with relatively low maximum luminance (up to about $100 \mathrm{~cd} / \mathrm{m}^{2}$ ), but more accurate functions have been proposed for perceptual linearization of the input signal in brighter High Dynamic Range (HDR) displays [7].

\subsection{Pixel compensation and clipping}

When backlight dimming is performed, some pixels receive less light than with full backlight. In many cases, this can be compensated by increasing pixel transmittance accordingly. This is referred to as pixel compensation [9] or brightness preservation [6]. In an ideal case, all the pixels can obtain their original intended values $T_{O R G}(i, j)$ (i.e. transmittance assuming full backlight) via compensation; in this case, the backlight dimming algorithm is said to be clipper-free. Then, the compensated transmittance $T_{\text {СОM }}(i, j)$ can be computed as:

$$
T_{C O M}(i, j)=\frac{T_{O R G}(i, j)}{B(i, j)}
$$

However, it might be desirable to allow clipping. Especially when there are only few bright pixels in the image, the image quality may not be severely compromised if those pixels are dimmer than in the original.

There are two basic approaches for clipping: hard and soft clipping [10]. In hard clipping, all the pixels that need to be clipped, are compensated to full transmittance. Hard clipping minimizes the average difference between originally intended and clipped light intensity, but on the other hand, it maps all the pixels above clipping threshold into one intensity level, causing undesirable saturation effect. This problem can be alleviated by using soft clipping, where the clipping curve is smoothened so that some level of differentiation in saturated areas can be obtained.

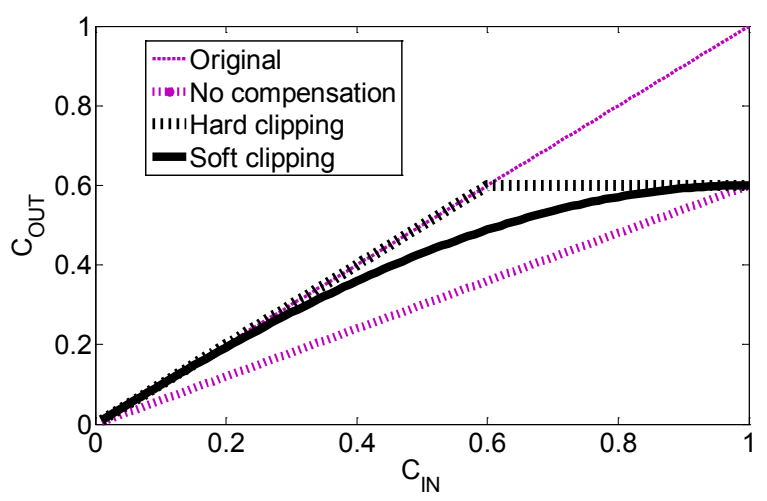

Fig. 2. Hard and soft clipping compared.

Hard and soft clipping are illustrated in Fig. 2. A practical example demonstrating the clipping effect is shown in Fig. 3. In this example, backlight of the original image (a) is dimmed by setting global backlight level to 0.325 , respectively to 0.6 in perceptual domain. The resulting images are shown without pixel compensation (b), with hard clipping (c), and soft clipping (d). For soft clipping, we use scaling function:

$$
C_{\text {OUT }}=C_{I N}-(1-B) \cdot C_{I N}^{1 /(1-B)}
$$

where $C_{I N}$ and $C_{\text {OUT }}$ are the original and rescaled pixel values, respectively. The same equation applies to $R, G$, and B components. Note that this is just one possibility to implement soft clipping: different approaches have been proposed in the literature [10].

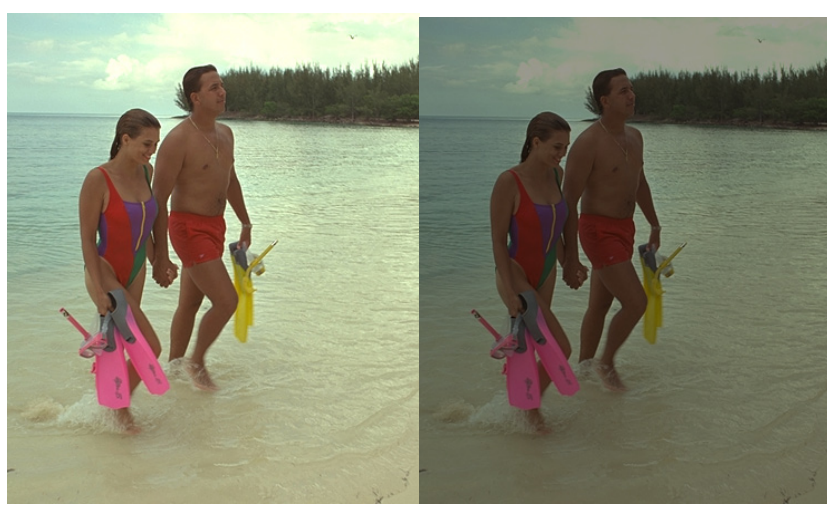

a) original image

b) no compensation

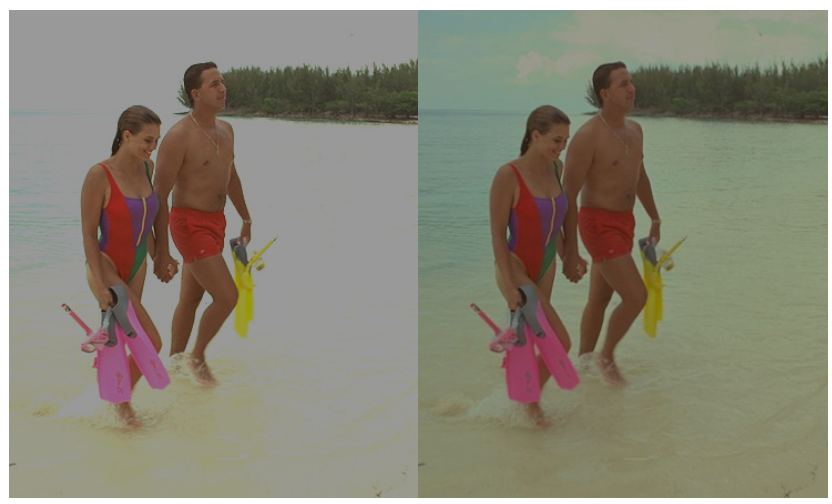

c) hard clipping

d) soft clipping

Fig. 3. Practical example of dimmed images without pixel compensation (b), hard clipping (c) and soft clipping (d).

As the visual inspection of Fig. 3. reveals, the relative differences between tones are maintained best when pixel compensation is not used, but the resulting image is very dark. On the other hand, hard clipping introduces visible saturation and color distortion in the brightest areas of the image. Among these compensation strategies, soft clipping apparently provides the most pleasant visual experience: the image looks brighter than without compensation, and colors are less distorted than with hard clipping.

The pixel compensation paradigm can also be applied reversely to compensate light leakage (i.e. reduce 
transmittance of dark pixels). That said, the impact of leakage is most typically visible only in the darkest segments, and this is why light leakage compensation is usually needed in the very lowest part of the luminance range only. In the example scenario above, light leakage is omitted.

\section{OBJECTIVE QUALITY MODEL}

In order to assess the quality of an image shown on an LCD employing backlight dimming and compare different backlight dimming algorithms, we may apply the model presented in Section 2 to generate a digital image that approximates the image actually shown on the screen in the perceptually uniform space, $L_{U}(i, j)$. Then, the resulting image or video sequence can be compared against the original sRGB input image [7], using any traditional objective quality metric, such as PSNR or Mean Structural Similarity Index Metric (MSSIM). The procedure is outlined in Fig. 4. For the sake of clarity, we assume that the signal is normalized to range $[0,1]$ in all phases.

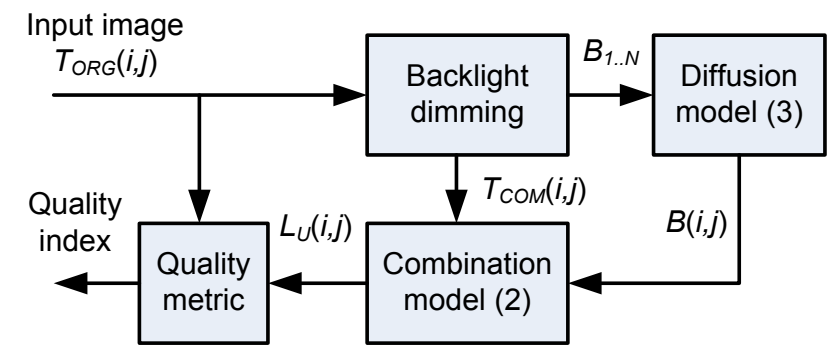

Fig. 4. Measuring the image quality in a backlight dimming systems.

The major weakness of the approach described above is that it does not consider the typical artifacts related to backlight dimming, such as color distortions, often difficult to estimate with traditional quality indices weighting different color components in a non-optimal manner. This is why we propose a metric based on PSNR, but operating in the CIE $\mathrm{L}^{*} \mathrm{a} \mathrm{b}^{*}$ color space, where $\mathrm{L}^{*}$ component represents luminance, and $\mathrm{a}^{*}$ and $\mathrm{b}^{*}$ components define the chrominance. The formula for conversion from linearized (Gamma corrected) sRGB to L*a*b* can be found in [9].

In contrast to other well known color spaces, L*a*b* color space has been designed so that a transition of certain magnitude in the color space is supposed to approximate an equivalent perceived change, regardless of the direction. This is why we can define color difference $\Delta E^{*}$, that is the perceived difference between two colors, considering both luminance and chrominance differences [9]:

$$
\Delta E^{*}=\sqrt{\left(\Delta L^{*}\right)^{2}+\left(\Delta a^{*}\right)^{2}+\left(\Delta b^{*}\right)^{2}},
$$

where $\Delta L^{*}, \Delta a^{*}$, and $\Delta b^{*}$ are the differences of $L^{*}, a^{*}$ and $b^{*}$ components between the compared colors. Using the well known definition of PSNR, we can define LabPSNR by replacing the Mean Squared Error (MSE) by the mean squared $\Delta E^{*}$ :

$$
\operatorname{LabPSNR}=10 \cdot \log _{10}\left(\frac{\left(\Delta E_{\text {max }}^{*}\right)^{2}}{\frac{1}{m n} \sum_{i=1}^{n} \sum_{j=1}^{m} \Delta E^{*}(i, j)^{2}}\right)
$$

where $\Delta E^{*}(i, j)$ is the color difference at pixel position $(i, j)$, $m$ and $n$ give the image dimensions, and $\Delta E^{*}{ }_{\max }$ is the maximum possible color difference. However, as $\Delta E^{*}{ }_{\text {max }}$ we have used $\Delta E^{*}$ obtained between normalized sRGB triplets $(0,0,0)$ and $(1,1,1)$, ie. the difference between full black and white, although even larger differences can be observed with certain color combinations. The value of $\Delta E^{*}{ }_{\max }$ is 100 .

In order to perform more accurate transform of the sRGB color component value $C_{U}$ in the normalized perceptually uniform scale to the respective normalized physical luminance value $C_{P H Y}$ than with the conventional Gamma function, we propose linearization using the conversion function adopted from [7]:

$$
C_{P H Y}=\frac{\left(\left(\exp \left[C_{U} \cdot \ln \left(a \cdot l_{\max }^{b}+1\right)\right]-1\right) / a\right)^{1 / b}}{l_{\max }},
$$

where $l_{\max }$ is the maximum physical luminance in $\mathrm{cd} / \mathrm{m}^{2}$, respective to $C_{U}=C_{P H Y}=1$. Parameters $a=0.56$ and $b=0.88$ have been derived by fitting Eq. (9) to the experimental data from [8], as explained in [7]. The reverse conversion can be performed as:

$$
C_{U}=\frac{\ln \left(a \cdot\left(l_{\max }^{b} \cdot C_{P H Y}\right)^{b}+1\right)}{\ln \left(a \cdot l_{\max }^{b}+1\right)}
$$

The use of the proposed function for perceptual linearization is of course optional; the conventional Gamma function can be used as well. However, it is essential that both original reference image and the distorted image are linearized with the same method.

One significant issue to consider for practical quality assessment is the selection of the luminance range for the reference image. Usually, the range is chosen so that a white pixel, i.e. a pixel with normalized sRGB triplet values $(1,1,1)$, is mapped to the peak luminance that can be obtained using the full backlight. However, with this approach, hard clipping results in better measured quality than soft clipping, and the quality impact of the saturation effect related to hard clipping is not taken into consideration. It is reasonable to assume that the relative luminance differences are as essential for the perceived quality as the maximum brightness level. This is why we also suggest rescaling the reference pixels to make the maximum obtained luminance to match the reference value:

$$
C_{R S}=\delta \cdot C_{R E F}
$$

where $C_{R S}$ is the rescaled pixel, $C_{R E F}$ is the respective original reference pixel, and $\delta$ is a linear dimming factor 
denoting the maximum observed relative loss of brightness after backlight dimming. The rescaled pixels represent the brightest possible dimmed image that would maintain the color uniformity of the original image. In general, $\delta=\min \left\{C_{D I M} / C_{R E F}\right\}$ can be used, where $C_{D I M}$ denotes the pixel values resulting from backlight dimming and pixel compensation. With global dimming, the minimum is typically reached at any position, where one of the brightest pixels is located. However, since local dimming can lead to significant local variations in the backlight level, the impact of outlier pixels with little significance on the overall image quality needs to be excluded. In this paper, we have used 1000 pixels out of the reference pixels with original intensity higher than $95 \%$ of the observed maximum intensity, so that they give the smallest $C_{D I M} / C_{R E F}$ values, and computed $\delta$ as the average of them. The rescaling procedure is then performed to the pixel values in the perceptually uniform domain. The procedure is illustrated in Fig. 5.

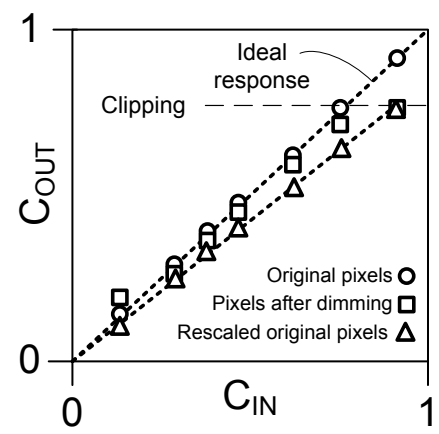

Fig. 5. Rescaling of the reference pixels.

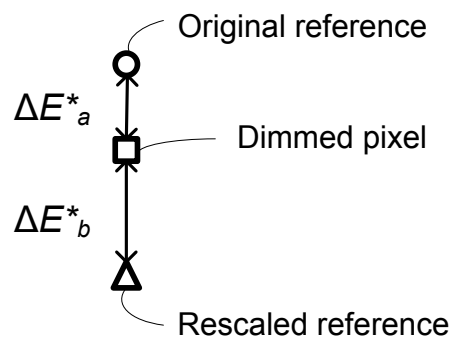

Fig. 6. Deriving $\Delta E^{*}$ from the difference between the dimmed pixel, and the original and rescaled pixels.

Even though the relative luminance levels are perceptually more important than the maximum brightness of the image, overall dimming has some negative influence on the quality. This is why we have the redefined color difference $\Delta E^{*}$ for each pixel so that the difference from the dimmed pixel is computed firstly against the original and secondly against the rescaled reference pixels, and the larger difference is chosen as $\Delta E^{*}$ :

$$
\Delta E^{*}=\max \left(\Delta E^{*}{ }_{a}, \Delta E^{*}{ }_{b}\right)
$$

Then, combined LabPSNR (CLabPSNR) can be computed, using $\Delta E^{*}$ from Eq. (14). We believe that this approach provides a reasonable compromise between measuring uniformity and brightness in respect to the ideal pixel value.

\section{EXPERIMENTS}

We have first tested our metric by simulating global backlight dimming on selected color images of size $768 \times 512$ pixels from the Kodak image set, using several backlight levels and different pixel compensation methods in a similar fashion as explained in Section 2.3. As expected, conventional RGB-PSNR indicates the best quality when hard clipping is used, since hard clipping minimizes the absolute difference between the pixel values in the original and the dimmed image. The relative quality estimates produced by the proposed CLabPSNR are better in line with the visually perceived image quality: as demonstrated in Fig. 3, soft clipping gives the best result, whereas hard clipping and direct scaling (no compensation) both show lower quality. As an example, RGB-PSNR and CLabPSNR results for the 'Beach' image shown in Fig. 3. are summarized in Table 1.

Table 1. Comparison of RGB-PSNR and CLabPSNR on Beach image with different pixel compensation (see Fig. 3.)

\begin{tabular}{lccc}
\hline & no comp. & hard clip. & soft clip. \\
\hline RGB-PSNR (dB) & 12.02 & $\mathbf{1 9 . 3 4}$ & 15.67 \\
\hline CLabPSNR (dB) & 11.41 & 13.13 & $\mathbf{1 4 . 9 7}$ \\
\hline
\end{tabular}

In order to validate CLabPSNR in local backlight dimming scenarios with more subtle differences between different backlight dimming methods, we have compared the proposed method against the raw subjective data available from an earlier study described in [11,12]. In this study, the subjective quality produced by different local backlight dimming methods was evaluated by 16 test subjects via pairwise comparisons. The comparison results were then converted into subjective scores using ThurstoneMosteller method [13]. Five to eight different backlight dimming methods were applied to each of the 7 source images included in the study. For more details about the subjective test methodology and the results, readers may refer to $[11,12]$.

Table 2. summarizes the results in terms of Pearson correlation coefficient (PCC) and Spearman rank order correlation coefficient (SROCC), when subjective results are compared against CLabPSNR and some conventional quality indices, including RGB-PSNR. Since only few versions of each test image were compared, the results are sensitive to outliers, which explains the low correlations in some cases, such as MSSIM and RGB-PSNR for 'Stars' image. 
Table 2. Comparison of CLabPSNR and other metrics using raw subjective results from the experiment $[11,12]$.

\begin{tabular}{|c|c|c|c|c|c|c|c|c|c|c|c|c|c|c|c|c|}
\hline & \multicolumn{2}{|c|}{ Stars } & \multicolumn{2}{|c|}{ Beach } & \multicolumn{2}{|c|}{ Parrot } & \multicolumn{2}{|c|}{ Volcano } & \multicolumn{2}{|c|}{ Diver } & \multicolumn{2}{|c|}{ Flower } & \multicolumn{2}{|c|}{ Lizard } & \multicolumn{2}{|c|}{$\overline{\text { All }}$} \\
\hline & PCC & SROCC & PCC & SROCC & PCC & SROCC & PCC & SROCC & PCC & SROCC & PCC & SROCC & PCC & SROCC & PCC & SROCC \\
\hline RGB-PSNR & 0.29 & 0.10 & 0.98 & 0.86 & 0.99 & 0.93 & 0.99 & 0.93 & 0.82 & 0.83 & 0.95 & 1.00 & 0.97 & 1.00 & 0.81 & 0.87 \\
\hline CLabPSNR & 0.95 & 0.90 & 0.98 & 0.86 & 0.96 & 0.93 & 0.96 & 0.93 & 0.75 & 0.66 & 0.96 & 0.98 & 0.98 & 0.96 & 0.88 & 0.87 \\
\hline MSSIM & 0.37 & 0.40 & 0.96 & 0.86 & 0.91 & 0.98 & 0.14 & 0.18 & 0.89 & 0.94 & 0.81 & 0.98 & 0.85 & 0.96 & 0.31 & 0.59 \\
\hline HDR-VDP & 0.77 & 0.80 & 0.97 & $\overline{0.86}$ & 0.93 & 0.98 & 0.96 & $\overline{0.96}$ & 0.82 & 0.83 & 0.73 & 0.90 & 0.90 & 0.96 & 0.82 & 0.81 \\
\hline PSNR-HVS-M & 0.27 & 0.10 & 0.98 & 0.86 & 0.99 & 0.93 & 0.93 & 0.86 & 0.84 & 0.89 & 0.97 & 0.98 & 0.97 & 1.00 & 0.78 & 0.78 \\
\hline
\end{tabular}

With the notable exceptions of 'Stars' and 'Diver', both RGB-PSNR and CLabPSNR show a good performance. When correlation coefficients are computed for all the images combined, the advantage of using CLabPSNR is more clearly pronounced, since PCC is significantly higher. We have also computed combined correlation coefficients with 'Stars' and 'Diver' excluded as outliers due to large difference between RGB-PSNR and CLabPSNR results, and CLabPSNR performs still better (for RGB-PSNR, $\mathrm{PCC}=0.83$ and $\mathrm{SROCC}=0.85$; for $\mathrm{CLabPSNR}, \mathrm{PCC}=0.92$ and $\mathrm{SROCC}=0.94)$. Therefore, the results clearly indicate that CLabPSNR has more consistent performance than RGB-PSNR. HDR-VDP also gives relatively consistent results across different contents, but it is also significantly more complex metric than CLabPSNR.

To our knowledge, CLabPSNR is the first attempt to develop an image quality metric that considers clipping and color distortions that are typical to local backlight dimming displays. In the future, our intention is to continue development towards a more accurate metric exploiting even more sophisticated features of the human visual system in the context of local backlight dimming.

\section{CONCLUSIONS}

In this paper, we have studied objective image quality assessment in a simulated backlight dimming system. We have observed that conventional quality analysis based on MSE or PSNR comparison between the original sRGB image and the image generated by simulating backlight dimming does not consider the impact of color distortion and pixel compensation adequately. Therefore, we have proposed a new metric, based on PSNR computation in $\mathrm{L} * \mathrm{a} * \mathrm{~b} *$ color space. The reference pixels are chosen from the original reference image, representing target image at full backlight, and linearly rescaled reference image, representing dimmed image that maintains the relative uniformity of the pixel values.

Informal evaluation of images produced by simulating global dimming with different clipping strategies suggest that the proposed metric approximates the perceived relative image quality more accurately than conventional PSNR comparing reference image and the dimmed image directly. In addition, we have used subjective results from our previous study comparing different local backlight dimming algorithms. The results show that CLabPSNR predicts subjective preferences more accurately than conventional PSNR and even more complex metrics, such HDR-VDP.

\section{REFERENCES}

[1] H. Groot Hulze and P. de Greef, "Power Savings by Local Dimming on a LCD Panel with Side Lit Backlight," SID Symp. Digest of Technical Papers, vol. 40, no. 1, pp. 749-752, Jun. 2009.

[2] H. Cho and O.-K. Kwon, "A Backlight Dimming Algorithm for Low Power and High Image Quality LCD Applications," IEEE Trans. Consumer Electr., vol. 55, no. 2, pp. 839-844, May 2009.

[3] H. Nam, "Low Power Active Dimming Liquid Crystal Display with High Resolution Backlight," Electronics Letters, vol. 47, no. 9, pp. 538-540, Apr. 2011.

[4] M. Albrecht, A. Karrenbauer, T. Jung, and C. Xu, "Sorted Sector Covering Combined with Image Condensation-An Efficient Method for Local Dimming of Direct-Lit and Edge-Lit LCDs," IEICE Trans. Electr., vol. 93, no. 11, pp. 1556-1563, Nov. 2010.

[5] X. Shu, X. Wu, and S. Forchhammer, "Optimal Local Dimming for LED-backlit LCD Displays via Linear Programming," in Proc. SPIE EI, vol. 8305, Jan. 2012.

[6] N. Burini, E. Nadernejad, J. Korhonen, S. Forchhammer, and $\mathrm{X}$. Wu, "Image-Dependent Energy-Constrained Local Backlight Dimming," in Proc. ICIP, Orlando, FL, USA, Sep. 2012.

[7] J. Korhonen, N. Burini, S. Forchhammer, and J. M. Pedersen, "Modeling LCD displays with local backlight dimming for image quality assessment," in Proc. SPIE EI, vol. 7866, Jan. 2011.

[8] T. Aydın, R.Mantiuk, and H-P.Seidel, "Extending Quality Metrics to Full Luminance Range Images," in Proc. SPIE EI, vol. 6806, Jan. 2008.

[9] H. Nam, "A Color Compensation Algorithm to Avoid Color Distortion in Active Dimming Liquid Crystal Displays," IEEE Trans. Consumer Electr., vol. 56, no. 4, pp. 2569-2576, Nov. 2010.

[10] L. Kerofsky, and S. Daly, "Brightness Preservation for LCD Backlight Dimming," J. of the SID, 14/12, pp. 1111-1118, 2006.

[11] E. Nadernejad, N. Burini, J. Korhonen, S. Forchhammer, and C. Mantel, "Adaptive Local Backlight Dimming Algorithm based on Local Histogram and Image Characteristics," in Proc. SPIE EI, vol. 8652, Jan. 2013.

[12] C. Mantel, N. Burini, E. Nadernejad, J. Korhonen, and S. Forchhammer, "Quality Assessment of Images Displayed on LCD Screen with Local Backlight Dimming," in Proc. QoMEX, Klagenfurt, Austria, Jul. 2013.

[13] J. C. Handley, "Comparative Analysis of Bradley-Terry and Thurstone-Mosteller Paired Comparison Models for Image Quality Assessment," in Proc. PICS, Montreal, Canada, Apr. 2001. 